\title{
An Approach to Human Adaptability towards its Built Environment: A Review
}

\author{
Richa Tiwari ${ }^{1 *}$, Mukesh Pandey ${ }^{2}$, Anupama Sharma ${ }^{3}$ \\ ${ }^{1}$ Architecture, SPA, Bhopal, India \\ ${ }^{2}$ Deptartment of Mechanical Engineering, University Institute of Technology, Bhopal, India \\ ${ }^{3}$ Architecture \& Planning MANIT, Bhopal, India \\ E-mail:richatiwari@rediffmail.com,anukhurasia@yahoo.com \\ Received December 11, 2009; revised February 1, 2010; accepted March 17, 2010
}

\begin{abstract}
This paper deals with the human adaptability to its built environment. The built environment as we know it rarely finds itself adapting to its surrounding context, whether it be on the level of interaction with humans or the climate. Humans and nature both are in a constant state of flux; moving, changing, sensing, and reacting to their context and information they gather and perceive. A barrier is formed between the built environment and humans and nature due to the fact that their inherent characteristics are utterly contrasting. It is commonly estimated that persons in urban areas spend at least $80 \%$ of their time indoors. This suggests that the quality of the indoor environment can have a significant impact on comfort, health, and overall sense of well being. The indoor environment of buildings should thus be designed and controlled, as to provide a comfortable and healthy space for occupants. In order to maintain the quality of the indoor environment, we mechanically condition our buildings to achieve constant, uniform and comfortable environments. The maintenance of thermal equilibrium between the human body and its environment is one of the primary requirements. History of thermal comfort and climate design shows a definite relation between them and research is needed to know "What are comfort conditions?" and "How buildings could adapt themselves to these conditions".
\end{abstract}

Keywords: Adaptability, Human Comfort, Thermal Comfort, Thermal Performance, Adaptive Behaviour

\section{Introduction}

The phrase built environment refers to the man-made surroundings that provide the setting for human activity, ranging in scale from personal shelter to neighborhoods to the large-scale civic surroundings. Humans and many other mammals have unusually efficient internal temperature regulating systems that automatically maintain stable core body temperatures in cold winters and warm summers. In addition, people have developed cultural patterns and technologies that help them adjust to extremes of temperature and humidity.

Thermal comfort is essentially a subjective response. Current comfort standards such as ASHRAE-55 (American Society of Heating, Refrigerating and Air-Conditioning) [1] define thermal comfort as "state of mind, which expresses satisfaction with the thermal environment". While it may be partially influenced by a variety of contextual and cultural factors, a person's sense of thermal is primarily a result of the body's heat exchange with the environment. This is influenced by four parameters that constitute the thermal environment (Airtemperature, Radiant temperature, Humidity and Air speed) two personal parameters (Clothing and Activity level, or Metabolic rate) [2].

\section{Thermal Adaptation}

Environment and behaviour research teaches that ones experience of a place is a multi-variation phenomena and a reflection of the degree to which the place contributes to a person's objectives and expectations. In the adaptive approach to modeling-thermal comfort, thermal perception is affected by circumstances beyond the physics of the body's heat-balance, such as climate setting, social conditioning, economic considerations and other contextual factors [3]. The adaptive hypothesis states that one's satisfaction is achieved by matching the actual thermal environmental conditions prevailing at that point in time and space, with one's thermal expectations of what the 
indoor climate should be like. A variable temperature standard links indoor temperatures to the climatic context of the building and accounts for thermal experiences and current thermal expectations of their occupants. In short, satisfaction occurs through appropriate adaptation to the indoor climatic environment.

\section{Adaptive Behaviour}

An alternative to traditional comfort theory-termed the "adaptative" can broadly be interpreted as the gradual diminution of the organism's response to repeated environmental stimulation which building occupants undergo in order to improve the "fit" of the indoor climate to their personal requirements [3]. This is achieved either through the way they interact with the environment, or modify their own behaviour, or because contextual factors and past thermal history change their expectations and thermal preferences. It is possible to distinguish three modes of the adaptive behaviour as shown in Figure 1.

\subsection{Behavioural Adjustment}

"Behavioural thermoregulation is well developed in humans and becomes preponderant and tends to supplant other forms of thermoregulation". In this a person makes modifications consciously or unconsciously, which in turn will modify heat and mass fluxes governing the body's thermal balance. This modifications or adjustments can be defined in terms of three categories: Personal adjustments e.g. changing personal variables like clothing, activity etc. and adjusting to the surroundings. Technological adjustments e.g. modifying the surroundings themselves like turning on fans or opening or closing of windows etc. Cultural adjustments, which include scheduling activities or adapting, dress codes etc.

\subsection{Physiological Adjustments}

Physiological adjustments include changes in the physiological responses that result from exposure to thermal environmental factors leading to gradual diminution in the strain induced by such exposure. These adjustments can be sub categorized: Genetic adaptation, including alterations, which become part of the genetic heritage of an individual. Acclimatization includes changes in the settings of the physiological thermoregulation system over a period of time. Physiological acclimatization is mediated by the autonomic nervous system and directly affects the physiological thermoregulation set points.

\subsection{Psychological Adjustments}

Psychological adjustments refer to perceptual adaptation and encompass the effects of cognitive and cultural

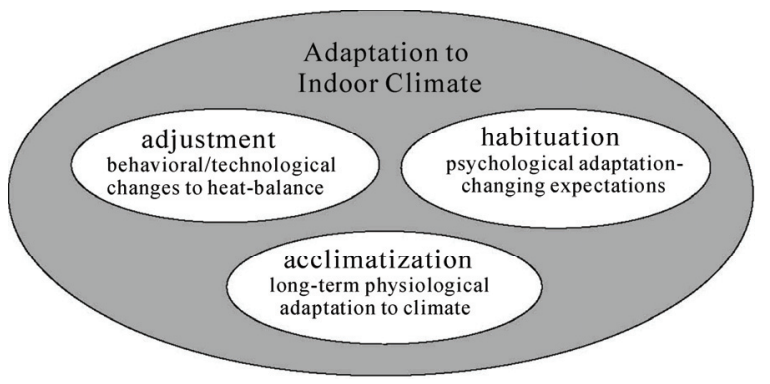

Figure 1. The three components of adaptation to indoor climate.

variables and describe the extent to which habituation and expectation alter one's perception of and reaction to sensory information due to past experiences and expectations [4].

The role of expectation in thermal comfort research was acknowledged in the earlier works of McIntyre, who stated that "a person's reaction to a temperature which is less than perfect will depend very much on his expectations, personality and what work he is doing at the time". Although the studies explain the differences in observed and predicted thermal sensations and acceptability, particularly of different environmental contexts such as the home vs office, or when comparing responses in airconditioned vs naturally ventilated buildings. However evidences shows that building occupants become accustomed to levels of warmth prevailing within buildings on time scales of weeks or months. These scales translate into synoptic and seasonal processes operating in the outdoor atmospheric environment.

An important premise of the adaptive model is that the building occupant is no longer simply a passive recipient of the thermal environment as given, as in the case of a climate chamber experimental subject, but instead is an active agent interacting with all levels of the person-environment system via feedback loops. The adaptive hypothesis indicates that one's satisfaction with an indoor climate is achieved by a correct matching between the actual thermal environmental conditions prevailing at that point in time and space, and one's thermal expectations of what the indoor climate should be like. Thermal expectations result from a confluence of current and past thermal experiences, cultural and technical practices $[3,5]$. These relationships have been described in Figure 2, a schematic diagram developed by $\mathrm{Au}-$ liciems [5] showing that a given set of indoor climatic conditions can elicit varying levels of comfort and satisfaction from building occupants, depending on culture or climatic and HVAC/architectural expectations.

\section{Field Evidences for Human Adaptation}

In recent years numbers of studies have been conducted on climate oriented building design to enhance 


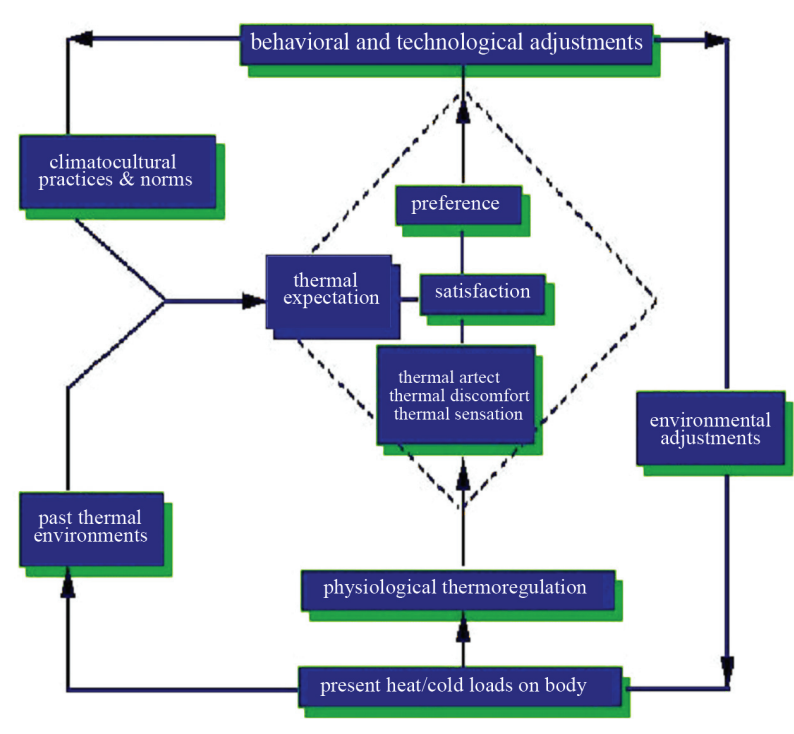

Figure 2. Schematic diagrams of thermal expectations.

thermal comfort conditions in living space and at the same time to reduce both the embodied and operational energy consumption. But results reported in these studies often deviate from the actual scenario. This discrepancy arises because most of the available thermal comfort standards are suited for air-conditioned buildings the situation leads to difficulty in estimating the thermal environments due to lack of adequate field experiments and long term data collection. In naturally ventilated buildings the occupants ability to modify the indoor environment is limited .An uncomfortable indoor environment might not be possible to control by passive means because of different socio-economic background and climatic condition. However, people living in naturally ventilated buildings are likely to be more tolerant.

Field studies were conducted for assessing the potential impacts of behavioural or psychological adaptations. Questionnaire based subjective measurements for this study was carried out. The respondents were asked to vote on ASHRAE 7 point thermal sensation scale followed by extensive interaction and filling up the questionnaire. This interaction helped us to record the common behavioural adaptations. Studies showed that, by utilizing behavioural adjustments such as wearing light clothing and restraining physical activities, local occupants were able to reach acceptable comfort in what-to immigrants from cold climates could be quite uncomfortable. The influence of clothing adjustments as a part of a study of office workers in naturally ventilated buildings in central India was also studied and it was found that the office occupants were comfortable across a wide range of seasonal temperatures, with neutralities varying between $15.7^{\circ} \mathrm{C}$. in winter and $26.4^{\circ} \mathrm{C}$ in summer. This concludes that at least two-thirds of the seasonal changes in comfort temperatures could be attributed to the flexibility in the traditional Indian clothing worn. While this supports the hypothesis that people use clothing adjustments to adapt themselves to a wide range of temperatures, it also suggests that there may be additional non-behavioural ways in which people adapt to the built environment.
ASHRAE Scale

$$
\begin{aligned}
& \cdot+3 \text { Hot } \\
& \cdot+2 \text { Warm } \\
& \cdot+1 \text { slightly warm } \\
& -0 \text { Neutral } \\
& --1 \text { slightly cool } \\
& --2 \text { Cool } \\
& --3 \text { Cold }
\end{aligned}
$$

\section{Bedford scale}

7 Much too warm

\section{Too warm}

5 Comfortably warm

4 Comfortable

\section{(neither cool nor warm) \\ 3 Comfortably cool \\ 2 Too cool}

\section{Much too cool}

In addition to adjusting oneself to the environment, one can also manipulate the environment itself. The occupants always try to provide themselves the thermal environment customary to their own socio-cultural context and local climate during the residential building design. Occupants of the house have a propensity to adapt to the changes in thermal environment and try to be more comfortable. The temperatures corresponding to comfortable thermal environment are not fixed but are continuing response to changes in both indoor and outdoor environmental condition modified by climate and social custom. Sudden changes in the ambient temperature imposed on the occupants actually lead to discomfort. Hourly questionnaires were used to study whether the subjects had made adjustments to their clothing or to their furniture, doors, windows, shades, fans or any other part of the building to improve their comfort. Results indicated extensive occupant interaction to adjustments to controls or other environmental aspects of the room and less adjustments to clothing. Benton and Brager [6] also conducted a field study of thermal comfort, which addressed the availability, use, and effectiveness of both personal and environmental behavioural adaptation.

The role of personal control on expectation and thermal response has important implications in naturally ventilated vs centrally air-conditioned buildings. The adaptive hypothesis implies that if occupants in a centrally-controlled building have generally experienced fairy constant and uniform conditions, with limited opportunities for personal control, then they not unreasonably expect their building to automatically provide them with perfect comfort. And when it fails to meet those expectations, they will be more likely to judge that building harshly compared to a situation were they had control over those conditions. Studies by Paciuk [7] show evidence of this, he found that personal or environmental adjustments in air-conditioned buildings actu- 
ally had a small effect on satisfaction. Studies were conducted in a mixed mode office building and it was found that, as temperatures rose above $24^{\circ} \mathrm{C}$, people in centrally air- conditioned work areas began voting much higher on the thermal sensation scale than their colleagues in the naturally ventilated work areas, suggesting that they were less tolerant of higher temperatures and expected a higher standard in the thermal environment. Studies also show the adaptive actions commonly occurring in offices and the percentage of people who choose to employ them. The main adaptive behaviours discussed in the study were opening windows and switching on a fan. The environmental control of opening windows is highly preferred by occupants. When people have the freedom to modify the environment and make the necessary adjustment, they use these actions to compensate for the less comfortable thermal conditions.

Similar patterns were found in thermal comfort field studies of homes vs. office buildings, where a multitude of contextual factors, including perceived control, might influence expectation and thermal response. It was found that thermal neutralities and preferences were significantly lower in the home compared to the offices and the differences could not be accounted for by changes in clothing, activity, or air velocity. All these patterns support the notion that people grow to accept the thermal conditions to which they become accustomed to and that this acceptance might be influenced by factors such as personal control, energy bills, or concern for the environment. In short, when people have the possibility to control their environment (e.g. by being able to open a window), they are more easily thermally satisfied than when they perceive that they do not have control. Satisfaction occurs through appropriate adaptation to the indoor climate environment.

Field evidence for thermal adaptation shows a clear distinction between the thermal adaptation and responses of occupants in naturally ventilated buildings as opposed to air-conditioned buildings. The survey also showed that this difference could not entirely be accounted for by adjustments to clothing or activity. The most plausible explanation for these differences is the contextual influence of thermal history and its effects on expectationspast thermal experiences in a building create a benchmark for expectations of future thermal performance. In naturally ventilated buildings, indoor temperatures more closely match the diurnal and seasonal variations in outdoor temperatures. People recognize this, relax their expectations or individual "comfort criteria" and not only become more tolerant of the more varied, dynamic and non-uniform indoor conditions, but often prefer having a closer connection with weather and seasonal changes. Comfort ultimately depends on the degree to which the environment matches and contributes to our expectations and studies have consistently shown that this is strongly affected by our sense of whether or not conditions are under control. All these patterns support the notion that people grow to accept the thermal conditions to which they become accustomed to and that this acceptance might be influenced by factors such as personal control, energy bills, or concern for the environment. In short, when people have the possibility to control their environment (e.g. by being able to open a window), they are more easily thermally satisfied than when they perceive that they do not have control. Satisfaction occurs through appropriate adaptation to the indoor climate environment.

\section{Thermal Performance of Buildings}

The adaptive approach is based on statistical analysis of large number of thermal comfort field studies. Field studies have more immediate relevance to living conditions. The adaptive approach is a behavioural approach and rests on the observation that people are not passive in relation to their environments, but they express direct response to make themselves comfortable at the given time and opportunity. The adaptive opportunity may be provided for instance by switching fans or operable windows or ventilators in summer or by temperature controls in winter. Since clothing and activity levels are region specific and driven by socio-cultural set-up and climate, it is very difficult to find a single value for comfort temperature. Different respondents vote according to their own physiological, psychological and behavioural adaptations. Because of this fact, it has been found that at same temperature: different respondents have different thermal sensation or same thermal sensation at different temperatures. An increasingly wide range of temperature is permissible as the adaptive opportunities increased. Individual control is more effective in advancing comfort than group control.

\section{Conclusions}

Expectation plays a role for occupants of air-conditioned buildings as well, but in a different way. Here, thermal history comprises consistently cool, constant, uniform conditions, creating more stringent comfort criteria while biasing expectations towards constant HVAC set points rather than daily or seasonal fluctuations. Air-conditioned occupants were basing their evaluations on the benchmark of their own preconceptions of what air-conditioning should achieve, rather than on what it actually provided. In effect, this suggests that increasing levels of sophistication in environmental control systems and building services are on a treadmill of attempting to satisfy increasingly stringent occupant expectations.

The adaptive processes are operating on time scales ranging from seasonal, through synoptic to diurnal. Critics of the adaptive approach at various symposia or se- 
minars have repeatedly asked the question: "how long must your people suffer in sub-optimal indoor climates before they become adapted?" The answer to this depends on which of the adaptive processes is being relied upon. While traditional research designs tend to look at responses at a given moment, experiments that intend to evaluate adaptive mechanisms need to take measurements over extended periods of time. Available evidence reviewed in this paper indicates that, in climate chamber experiments at least, the slower physiological adaptive process of acclimatization appears not to be relevant to this question of thermal neutrality and its fluctuations from day to day, week to week and season to season. As a result, the data analysis and model development will focus more heavily on the adaptive mechanisms of adjustment, and habituation/expectation.

One of the most important findings from our review of field evidence was the distinction between thermal comfort responses in air-conditioned vs. naturally ventilated buildings. Analysis suggested that behavioural adaptation incorporated in conventional heat balance models could only partially explain these differences and that comfort was significantly influenced by peoples expectations of the thermal environment. These contextual differences most likely resulted from a combination of past thermal history in buildings and differences in levels of perceived control. It is therefore essential that adaptive algorithms for comfort control utilize regressions from the architectural context for which they are intended. Regressions based on data from naturally ventilated buildings will probably be unsuitable as a control algorithm for air-conditioned buildings in which adaptive opportunities are severely constrained. There are numerous benefits to be gained from an improved understanding of the influence of adaptation on thermal com- fort in the built environment. Enhanced levels of thermal comfort and acceptability among occupants reduced energy consumption and the encouragement of climatically responsive building design. These benefits can best be achieved through an ongoing, open dialogue and collaboration between the proponents of the "adaptive" vs. "heat balance" approach and I hope that my paper has provided a function for that to occur.

\section{References}

[1] Thermal Environmental Conditions for Human Occupancy, American Society of Heating, Refrigerating and Air-Conditioning Engineers Standard 55-1992, Ashrae, Atlanta, 1992.

[2] A. B. Lovins, "Air-Conditioning Comfort: Behavioural and Cultural Issues," E source, Colorado, 1992.

[3] F. Nicol, "Thermal Comfort-A handbook for Field Studies towards an Adaptive Model," University of East London, UK, 1993.

[4] M. A. Humphreys, "Field Studies and Climate Chamber Experiments in Thermal Comfort Research. Thermal Comfort: Past, Present and Future," BRE, UK, 1994.

[5] A. Auliciems, "Thermal Comfort," In: N. Ruck, Ed., Building Design and Human Performance, Van Nostrand, NY, 1989.

[6] G. Brager, "A Global Database of Thermal Comfort Field Experiments," American Society of Heating, Refrigerating and Air-Conditioning Engineers Transactions, 1998.

[7] M. Paciuk, "The Role of Personal Control of the Environment in Thermal Comfort and Satisfaction at the Workplace," Doctoral Dissertation, University of Wisconsin, Milwaukee, 1989. 